\title{
Hausbesuche im Kindes- und Erwachsenenschutz in der Schweiz - eine qualitative Studie
}

\author{
Martina Koch • Markus Steffen • Rahel Bühler
}

Angenommen: 8. September 2020 / Online publiziert: 29. September 2020

(C) Der/die Autor(en) 2020

Zusammenfassung In dieser Forschungsnotiz wird ein laufendes Forschungsprojekt vorgestellt, das Hausbesuche im Kindes- und Erwachsenenschutz in verschiedenen Regionen der deutschsprachigen Schweiz untersucht. Für zwei Zeiträume (1960 bis 1980; 2000 bis 2020) wird rekonstruiert, aufgrund welcher fachlichen Überlegungen Hausbesuche bei Abklärungen von Gefährdungen im Kindes- und Erwachsenenschutz in Auftrag gegeben wurden/werden, wie sie abliefen/ablaufen, welches Wissen dabei produziert wurde/wird und wie dieses Wissen anschliessend prozessiert und entscheidungsrelevant gemacht wurde/wird. Die Untersuchung basiert auf einer qualitativen Analyse von Personenakten sowie auf einer Interviewstudie mit Fachkräften und von behördlichen Hausbesuchen betroffenen Menschen. Weiter werden teilnehmende Beobachtungen von Hausbesuchen durchgeführt.

Schlüsselwörter Hausbesuche $\cdot$ Kindes- und Erwachsenenschutz $\cdot$ Abklärungen von Gefährdungen · Aktenanalyse $\cdot$ Schweiz

Dr. M. Koch $(\bowtie) \cdot$ M. Steffen · R. Bühler

Hochschule für Soziale Arbeit, Fachhochschule Nordwestschweiz, Riggenbachstrasse 16, 4600 Olten,

Schweiz

E-Mail: martina.koch@fhnw.ch

M. Steffen

E-Mail: markus.steffen@fhnw.ch

R. Bühler

E-Mail: rahel.buehler@fhnw.ch 


\section{Home visits in child and adult protection in Switzerland: a qualitative research}

Abstract This research note presents an ongoing project investigating home visits in the field of child and adult protection in different regions in German-speaking Switzerland. The project combines a historical perspective on the period from 1960 to 1980 and a current perspective on the period from 2000 onwards. It is our goal to reconstruct on what professional considerations home visits were/are carried out and what knowledge was/is thereby produced. Furthermore, we analyse how home visits influence/d the processing of cases and the decision-making in the assessment of child and adult endangerment. The study is based on a qualitative analysis of case records as well as on an interview study with professionals and clients affected by home visits. In addition, we aim to explore home visits trough participant observations.

Keywords Home visits · Child protection - Adult protection · Risk assessments · Analysis of case records $\cdot$ Switzerland

\section{Hausbesuche in der Sozialen Arbeit - Einleitung}

Seit über hundert Jahren werden Hausbesuche in der Sozialen Arbeit für verschiedene Zwecke genutzt, z. B. um armutsbetroffene Familien und ihre Wohnungen zu inspizieren, um zu kontrollieren, wie sie ihren Haushalt führen und ihre Kinder erziehen, oder um Ansprüche auf materielle sowie immaterielle Hilfe abzuklären (z.B. Hancock und Pelton 1989; Matter 2011; Winter und Cree 2016). Auch heute noch finden Hausbesuche statt, inbesondere im Kindesschutz, wo sie gemeinhin als unumgänglich gelten (z. B. Ferguson 2016). Dennoch finden sich auch Stimmen, die auf die Grenzen von Hausbesuchen in diagnostischer Hinsicht sowie auf Risiken von Kontrollbesuchen für den Aufbau einer Hilfebeziehung verweisen (u. a. Meysen 2008) sowie Bedenken in grundrechtlicher Hinsicht äussern (u.a. Neuffer und Ollmann 2000). Vor dem Hintergrund eines möglichen Eingriffs in die Privatsphäre Betroffener erstaunt es, dass bisher international noch relativ wenig Forschung zu dieser Thematik vorliegt (Ausnahmen für Deutschland: Freres et al. 2019; UrbanStahl et al. 2017; Gerull 2014; sowie für England: Cook 2017, 2019; Ferguson 2016). In der Schweiz fehlen Studien, die Hausbesuche als eigenständiges Thema behandeln. 


\section{Hausbesuche als eigenständiger Forschungsgegenstand - Forschungsdesign}

Ein im Oktober 2018 an der Fachhochschule Nordwestschweiz gestartetes Forschungsprojekt ${ }^{1}$ erforscht abklärend-diagnostische Hausbesuche im Kindes- und Erwachsenenschutz in historischer (1960 bis 1980) sowie gegenwärtiger Perspektive (2000 bis 2020). Um dem föderalen Aufbau des Schweizer Kindes- und Erwachsenenschutzes gerecht zu werden, untersuchen wir verschiedene Regionen der Deutschschweiz. Aus sozialkonstruktivistischer Perspektive fragen wir danach, wie Hausbesuche stattfanden/stattfinden, welches Wissen dadurch erzeugt wurde/wird und wie dieses Wissen weiter prozessiert und entscheidungsrelevant gemacht wurde/ wird (vgl. auch Pomey 2017). Das empirisch-qualitative Forschungsdesign umfasst die Erhebung verschiedener Datenmaterialien: Sowohl das historische als auch das gegenwärtige Teilprojekt werten Fallakten aus, in denen sich Berichte von Hausbesuchen finden. Ferner führen wir insbesondere im gegenwärtigen Teilprojekt Expert*innen-Interviews mit Fachpersonen, die Hausbesuche in Auftrag geben oder durchführen und dokumentieren. Weiter ist geplant, auch von Hausbesuchen betroffene Menschen zu interviewen, um deren Sicht auf den Hausbesuch rekonstruieren zu können. Schliesslich führen wir teilnehmende Beobachtungen von Hausbesuchen durch. Wir werten alle Datenmaterialien mitels des dreistufigen Kodierverfahrens der Grounded Theory aus (Strauss und Corbin 1996).

\section{Hausbesuche an der Schnittstelle verschiedener Organisationen - Perspektiven und Erkenntnisinteressen}

In der Schweiz sind die lokalen Prozesse und Organisationsformen im Kindes- und Erwachsenenschutz vielfältig und wenig einheitlich. Mit der Abklärung wurden/ werden meistens Organisationen außerhalb der für die erstinstanzlichen Entscheidungen zuständigen Vormundschaftsbehörden (bis 2012) bzw. Kindes- und Erwachsenenschutzbehörden ( $a b$ 2013) mandatiert. Zum Zuge kamen und kommen dabei z. B. kommunale Sozialdienste, aber auch private Non-Profit- und Profit-Organisationen. Deshalb untersuchen wir Hausbesuche als behördlich induzierte Vorgänge, die häufig an der Schnittstelle zwischen mandatierender und mandatierter Organisation stattfanden/-finden. Wir interessieren uns dabei sowohl für Gemeinsamkeiten als auch für Unterschiede zwischen den Regionen und in der historischen Entwicklung: Wie wurden/werden Hausbesuche zwischen 1960 und 1980 und seit 2000 eingesetzt und wie wurden/werden sie dokumentiert? Wer ordnet(e) den Hausbesuch jeweils aus welchem Grund an? Welche fachlichen, juristischen, moralischnormativen etc. Erwägungen gingen/gehen dem Hausbesuch voran? Wie erleb(t)en betroffene Personen den Hausbesuch?

Diesen Fragen liegt die Annahme zugrunde, dass Akten den „Fall“ durch Praktiken des Schreibens mitkonstruieren (vgl. u.a. Pomey 2017, S. 85 f.). Akten sind

\footnotetext{
1 Das Projekt ist Teil des Nationalen Forschungsprogramms NFP 76 „Fürsorge und Zwang“ des Schweizerischen Nationalfonds (SNF) und läuft bis März 2022.
} 
jeweils ,aus dem Blickwinkel der Institution, die diese Akten für ihre Zwecke anlegt" (Galle und Meier 2009, S. 108), verfasst. Sie dienen der Legitimation sowie der Nachvollziehbarkeit der getroffenen Entscheide. Berichte von Hausbesuchen sind also kaum je ,neutrale“ Dokumente, denn sie sind in der Regel mit einem konkreten Auftrag und aus einer spezifischen Perspektive verfasst: Wie wurde/wird der „Fall““ durch Dokumentationen von Hausbesuchen mitkonstruiert und prozessiert? Wie beeinfluss(t)en Hausbesuche und dazugehörige Berichte den weiteren Fallverlauf, d.h. inwiefern spiel(t)en sie eine Rolle für nachfolgende behördliche Entscheidungen? Welcher Wandel bzw. welche Persistenzen zeigen sich in den Hausbesuchsberichten bezüglich normativer Annahmen, z. B. in Form abwertender Aussagen zu Personen und Wohnungen?

In der Analyse legen wir einen besonderen Fokus auf Geschlechtskonstruktionen im Kontext der Hausbesuchspraxen. Historische Befunde verweisen darauf, dass die Differenzkategorie Geschlecht in Hausbesuchsberichten eine grosse Rolle spielt (u.a. Businger und Ramsauer 2017; Matter 2011). Wie Studien zum Kindesschutz der Gegenwart zeigen, sind insbesondere Vorstellungen von ,guter Mutterschaft“ weiterhin von zentraler Bedeutung (u.a. Pomey 2017). Daher fragen wir: Welche geschlechtsspezifischen Beschreibungen bzw. Rollenerwartungen finden sich in den Berichten von Hausbesuchen und inwieweit haben sich diese seit 1960 gewandelt? Inwiefern beziehen sich Geschlechtskonstruktionen auf Wohnen und auf die Haushaltsführung?

Einen weiteren Fokus legen wir auf Raumkonstruktionen, womit wir auch die Grenze zwischen privat und öffentlich ausloten, die durch den Hausbesuch verschwimmt. Denn im „Hausbesuch [wird] der private Raum der Klient_innen zum öffentlichen Raum gemacht“ (Bastian und Schrödter 2014, S. 292; vgl. auch Wolff 1983). Hausbesuche bedürfen deshalb einer Aushandlung, ,wo die Grenze zwischen Arbeitsort und Wohnumgebung verläuft, aber auch, wie man sich an diesem Arbeitsort zu benehmen hat, der zugleich ein privater Wohnort ist" (Müller 2017, S. 299). Welche Vorstellungen von ,privat“ und „öffentlich“ lassen sich aus den Hausbesuchsberichten und den Interviews mit den betroffenen Personen sowie den Fachleuten rekonstruieren? Inwiefern verschwimmt die Grenze zwischen privat und öffentlich, welche Aushandlungen finden sich diesbezüglich?

$\mathrm{Zu}$ diesen Fragen sollten bis im Frühjahr 2022 die Ergebnisse unserer Studie vorliegen.

Funding Open access funding provided by FHNW University of Applied Sciences and Arts Northwestern Switzerland

Open Access Dieser Artikel wird unter der Creative Commons Namensnennung 4.0 International Lizenz veröffentlicht, welche die Nutzung, Vervielfältigung, Bearbeitung, Verbreitung und Wiedergabe in jeglichem Medium und Format erlaubt, sofern Sie den/die ursprünglichen Autor(en) und die Quelle ordnungsgemäß nennen, einen Link zur Creative Commons Lizenz beifügen und angeben, ob Änderungen vorgenommen wurden.

Die in diesem Artikel enthaltenen Bilder und sonstiges Drittmaterial unterliegen ebenfalls der genannten Creative Commons Lizenz, sofern sich aus der Abbildungslegende nichts anderes ergibt. Sofern das betreffende Material nicht unter der genannten Creative Commons Lizenz steht und die betreffende Handlung nicht nach gesetzlichen Vorschriften erlaubt ist, ist für die oben aufgeführten Weiterverwendungen des Materials die Einwilligung des jeweiligen Rechteinhabers einzuholen. 
Weitere Details zur Lizenz entnehmen Sie bitte der Lizenzinformation auf http://creativecommons.org/ licenses/by/4.0/deed.de.

\section{Literatur}

Bastian, P., \& Schrödter, M. (2014). Professionelle Urteilsbildung in der Sozialen Arbeit. Soziale Passagen, 6(2), 275-297.

Businger, S., \& Ramsauer, N. (2017). „Sie ist verschwenderisch und kann nicht sparen“ - Begründungen und Wissensproduktion in Vormundschaftsbehörden im Kanton Zürich in den 1950er und 1960er Jahren. In H. Messsmer (Hrsg.), Fallwissen. Wissensgebrauch in Praxiskontexten der Sozialen Arbeit (S. 23-48). Opladen: Barbara Budrich.

Cook, L.L. (2017). Making sense of the initial home visit: the role of intuition in child and family social workers' assessments of risk. Journal of Social Work Practice, 31(4), 431-444.

Cook, L.L. (2019). The home visit in child protection social work: emotion as resource and risk for professional judgement and practice. Child \& Family Social Work. https://doi.org/10.1111/cfs.12647.

Ferguson, H. (2016). Making home visits: creativity and embodied practices of home visiting in social work and child protection. Qualitative Social Work. https://doi.org/10.1177/1473325016656751.

Freres, K., Bastian, P., \& Schrödter, M. (2019). Jenseits von Fallverstehen und Prognostik - Wie Fachkräfte mit einer einfachen Heuristik verantworbaren Kindesschutz betreiben. Internationaler Forschungsüberblick und Befunde aus einer ethnographischen Studie zu Hausbesuchen durch das Jugendamt. neue Praxis, 2, 140-164.

Galle, S., \& Meier, T. (2009). Von Menschen und Akten. Die Aktion „Kinder der Landstrasse“ der Stiftung Pro Juventute. Zürich: Chronos.

Gerull, S. (2014). Hausbesuche in der Sozialen Arbeit. Eine arbeitsfeldübergreifende empirische Studie. Opladen: Barbara Budrich.

Hancock, B. L., \& Pelton, L. H. (1989). Home visits: history and functions. Social Casework, 70(1), 21-27.

Matter, S. (2011). Der Armut auf den Leib rücken. Die Professionalisierung der Sozialen Arbeit in der Schweiz (1900-1960). Zürich: Chronos.

Meysen, T. (2008). ,Ich war drin-Garantie” im Kinderschutz? Forum Erziehungshilfe, 14(4), 195.

Müller, F. (2017). Die „häusliche Umgebung“ als Wohnraum und Setting. Konflikte um Raumsouveränität in der ambulant-aufsuchenden Palliativversorgung. In M. Meuth (Hrsg.), Wohn-Räume und pädagogische Orte. Erziehungswissenschaftliche Zugänge zum Wohnen (S. 289-311). Wiesbaden: Springer.

Neuffer, M., \& Ollmann, R. (2000). Der Hausbesuch: Hausbesuche bei Gefährdung und Vernachlässigung von Kindern. Rechtsrahmen und fachlich-methodisches Vorgehen. Sozialmagazin, 25(9), 12-25.

Pomey, M. (2017). Vulnerabilität und Fremdunterbringung - Eine Studie zur Entscheidungspraxis bei Kindeswohlgefährdung. Weinheim: Beltz Juventa.

Strauss, A., \& Corbin, J. (1996). Grundlagen Qualitativer Sozialforschung. Weinheim: Beltz.

Urban-Stahl, U., Albrecht, M., \& Lattwein, S. (2017). Hausbesuche im Kinderschutz. Ergebnisse der Studie HabeK - Hausbesuche im Kontext des Schutzauftrags bei Kindeswohlgefährdung. Opladen: Barbara Budrich.

Winter, K., \& Cree, V.E. (2016). Social work home visits to children and families in the UK: a Foucauldian perspektive. British Journal of Social Work, 46(5), 1175-1190.

Wolff, S. (1983). Die Produktion von Fürsorglichkeit. Bielefeld: AJZ. 